\title{
Assessing the Open Trenches in Screening Railway Ground-Borne Vibrations by Means of Artificial Neural Network
}

\author{
Gaetano Di Mino, ${ }^{1}$ Marinella Giunta, ${ }^{2}$ and Ciro Maurizio Di Liberto ${ }^{1}$ \\ ${ }^{1}$ Department DIIV, University of Palermo, Parco D'Orleans, 90100 Palermo, Italy \\ ${ }^{2}$ Department DIMET, University Mediterranea of Reggio Calabria, Via Graziella Feo di Vito, 89100 Reggio Calabria, Italy
}

Correspondence should be addressed to Marinella Giunta, marinella.giunta@unirc.it

Received 29 January 2009; Revised 28 May 2009; Accepted 13 October 2009

Recommended by Mohammad Tawfik

Reducing ground borne vibrations in urban areas is a very challenging task in railway transportation. Many mitigation measures can be considered and applied; among these open trenches are very effective. This paper deals with the study of the effect, in terms of reduction of vertical and horizontal displacements and velocities, of the open trenches. 2D FEM simulations have been performed and several open trench configurations have been analysed varying the main geometric features such as width and depth, distance from the rail, thickness of the soil layer over the rigid bedrock, type of the ground, ratio between the depth of the trench, and the thickness of the soil layer. For quantifying the influence of the above specified parameters in reducing groundborne vibrations an analysis using artificial neural networks (ANNs) has been carried out. Results show that among the geometric parameters the role of the depth of the trench is very significant; however the influence of the depth must be also evaluated in relation to the thickness of the soil layer.

Copyright ( 2009 Gaetano Di Mino et al. This is an open access article distributed under the Creative Commons Attribution License, which permits unrestricted use, distribution, and reproduction in any medium, provided the original work is properly cited.

\section{Introduction}

The problem of the ground-borne vibrations induced by railway traffic has received an increasing interest in the recent years becoming a relevant scientific and technical research area.

Due to the frequent construction of high-speed railways and mass rapid transit systems worldwide, most highly developed cities or metropolitan areas have encountered the problem that the rail tracks inevitably come cross or close to vibration-sensitive sites, as discussed by Hung and Yang $[1,2]$.

The economical and environmental aspects of the issue require a careful assessment of the problem before the construction of new rail tracks or the upgrading of the existing ones for heavier and faster traffic.

The main source of excitation of the track is represented by the vertical force determined by the wheel-rail interaction during the passage of the train. Vibrations can be amplified by the passage of trains due to the surface irregularities of wheels and rails, by the rise and fall of axles over sleepers, and by the propagation of deformation patterns in the track and ground. Such vibrations are transmitted through the track structure, including rails, sleepers, ballast, and sublayers and propagate as waves through the soil medium [3].

The study of the ground-borne vibrations requires the consideration of four main components: the "source," that generate the vibration, that is, the excitation caused by the motion of the trains over rails with irregular surfaces, the "propagation path" through the soil medium, the "receiver," that is, the nearby buildings, and finally the "interceptor," that is, wave barriers, such as piles, in-filled and open trenches, and isolation pads.

At each component is related one specific phase of the process of the transmission of the vibrations and in particular the generation, the transmission, the reception, and the interception.

In regard to this last phase, three groups of mitigation measures can be considered:

(i) mitigations in the source, that include all types of interventions in the railway structures (active isolation); 
(ii) mitigations in the path, such as barriers to the waves propagation from the source to the receiver (in-filled and open trenches, lime cements columns); these barriers provide an active isolation when they are close to the source, and a passive one when they are far away;

(iii) mitigations to the receiver, that include all the measures aimed at reducing the effects on the buildings (passive isolation) or on the other vibration-sensitive sites.

Among the mitigation measures in the path, the open trenches have exhibited a good performance on the screening of the vibrations but, to achieve this effect, it is very important to assign it the proper dimensions.

\section{Previous Studies}

The first experimental surveys on the effectiveness of open and in-filled trenches were carried out by Barkam (1962) [4]. He showed that their effectiveness increases with increasing the depth and the distance for raising frequencies.

In regard to the continuous barriers also Woods [3] showed the influence of the depth and the distance from the vibratory source. He also demonstrated that the passive isolation is better than the active for screening the $P$ body and $S$-body waves while the active isolation is more suitable for the screening of the Rayleigh waves; moreover he highlighted that the most relevant geometric parameter in the screening process is the ratio between depth barrier and Rayleigh wavelength.

Several experimental surveys stated that the best screening performance takes place when the depth of the trench is equal to the wavelength while the width of the trench is small [3-10].

Numerous researches on the effectiveness of the barriers have been carried out using FEM and BEM modelling [1116].

In particular, Beskos et al. [11] employed boundary element method (BEM) to study open and in-filled trenches as well as pile wave barriers. Ahmad and Al-Hussaini [13] proposed a simplified design methodology for vibration screening of machine foundations by trenches using $2 \mathrm{D}$ BEM.

Adam and Estorff (2005) [6] inspected the effectiveness of open and in-filled trenches in reducing the six-storey building vibrations due to passing trains using a twodimensional FEM analysis.

Yang and Hung (1997) [1,2] combined the finite and infinite elements to investigate the effect of trenches and elastic foundations in reducing train-induced vibration.

Few works focus on the effectiveness of barriers in full scale.

The most important application is based on Gas Cushion Method that consists in a vertical panels filled of gas and flexible cushion with very low impedance installed in a trench having a great depth [17]. In Gnarp, Sweden 1984, the first application of these barriers was carried out in order to screen a sensitive building from railway ground borne

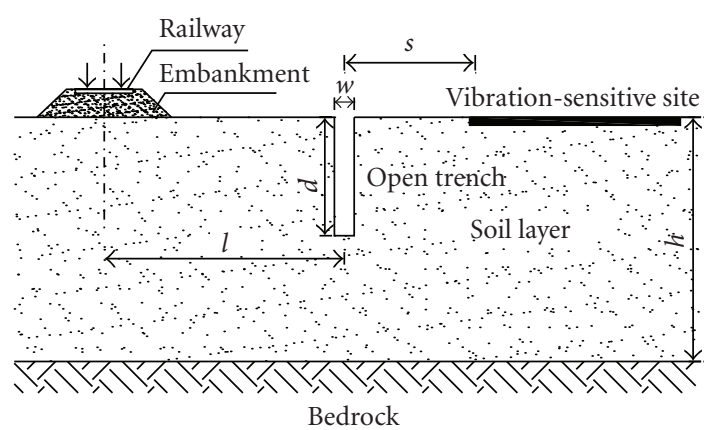

Figure 1: Schematic of the position of the open trench.

TABLE 1: Geometric parameters of the trenches.

\begin{tabular}{lcc}
\hline $\begin{array}{l}\text { Width } \\
w(\mathrm{~m})\end{array}$ & Distance from the rail $l(\mathrm{~m})$ & Distance from the site $s(\mathrm{~m})$ \\
\hline 0.5 & 8 & 19 \\
1.0 & 12 & 15 \\
1.5 & 16 & 11 \\
2.5 & 20 & 7 \\
- & 24 & 3 \\
\hline
\end{tabular}

TABLE 2: Depth of the trench and ratio depth/thickness of layer.

\begin{tabular}{lc}
\hline Parameters & Assumed values \\
\hline$d(\mathrm{~m})$ & $2,5,8,11,14$ \\
$d / h$ & $1 / 5,1 / 2,2 / 3$ \\
\hline
\end{tabular}

vibrations. The effectiveness of this application reached an attenuation value of about $70 \%$.

\section{Problem Formulation and Basic Assumptions}

In this work the screening performance of open trenches, excavated in a soil layer over a bedrock, has been studied. By applying a 2D FEM model, an extensive analysis has been carried out to the aim of determining the contribution of the main geometric parameters of the trenches in the interception of the vibrations. The geometric characteristics assumed to be variable parameters are width $(w)$, depth $(d)$, distance from the rail $(l)$, distance from the vibrationsensitive site $(s)$, thickness of the soil layer over the rigid base $(h)$, and ratio between the depth of the trench and the thickness of the soil layer $(d / h)$ (see Figure 1$)$.

The study has been carried out considering different scenarios obtained by varying the above specified geometric parameters of the trenches and the mechanical and geometric characteristics of the soil medium. The values attributed to the geometric features are summarised in Table 1, while the geometric conditions of the soil medium have been set up according to the Table 2 .

Two types of unsaturated soil, labelled, according to the classification of Eurocode 8, respectively, ground type C (medium dense sand) and ground type D (firm clay), have been considered. These soils have been characterised by the 


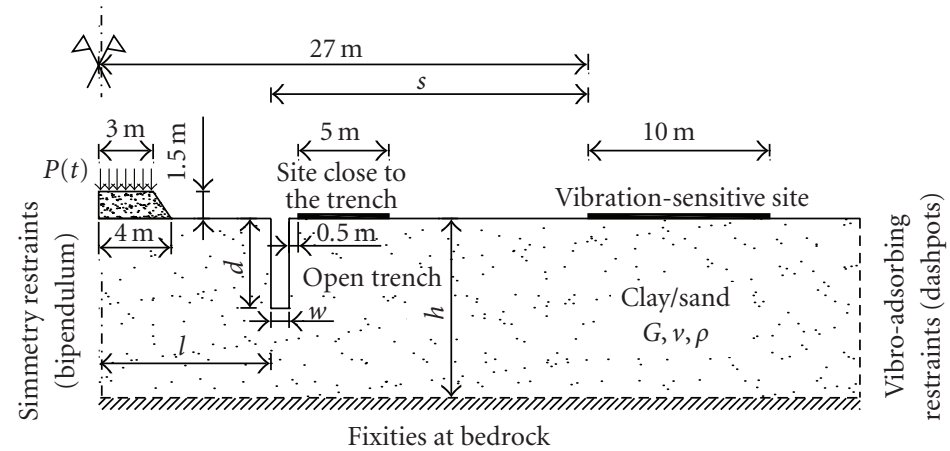

FIGURE 2: Modelling scheme.

shear modulus $(G)$, the Poisson's ratio $(\nu)$, the mass density $(\rho)$, the damping ratio $(\xi)$, and the shear and the Rayleigh's wave velocities $\left(V_{s}, V_{r}\right)$. The values assigned to each of these parameters are shown in Table 3.

The value of the damping ratio for clay and sand has been selected according to the results of an Italian comprehensive review [18] that analyses a great number of experimental results (44 international researches carried out in the last 30 years) and concludes that although a considerable scatter of the experimental points exists, for deformations lower than $0.001 \%$, the damping ratio has a tendency to increase according to the plasticity index.

By combining all the parameters, 540 scenarios have been obtained and investigated. The propagation and interception of ground-borne vibrations for each scenario have been modelled by means of the finite elements method. The scheme adopted is shown in Figure 2. The 2D model has been assumed to be symmetric in respect to the railway line.

It has been also established that the railway line is sited over an embankment $1.5 \mathrm{~m}$ high and with escarpment's slope of $2 / 3$; the geotechnical parameters of the embankment are $G=125 \mathrm{MPa}, \rho=2000 \mathrm{~N} / \mathrm{m}^{4} \mathrm{~s}^{2}$, and $\nu=0.33$.

For each scenario the following responses have been obtained: vertical (dir. $z$ ) and horizontal (dir. $y$ ) displacements and vertical (dir. $z$ ) and horizontal (dir. $y$ ) velocities. In particular, these parameters have been calculated in two areas:

(a) in a "vibration-sensitive site," large $10 \mathrm{~m}$ and far away $27 \mathrm{~m}$ from the symmetry axis of the railway;

(b) in a zone, in the following called "site close to the trench," $5 \mathrm{~m}$ wide and sited far away $0.5 \mathrm{~m}$ from the trench.

The dimensions assigned to the two sites are well suited to be comparable with the dimensions of receptors such us buildings.

The study of the response in this last site allowed to verify the effectiveness of the open trench as passive isolation.

\section{Finite Element Modelling}

The propagation of vibrations is a typical three-dimensional issue, specially in railway field where the train acts as a series of incoherent point source rather than a fully coherent line source. So three-dimensional models are certainly more suitable to predict the absolute vibration but require computation times not always consistent with extensive analyses.

Andersen and Jones [19] performed analyses 2D-3D FEM and BEM and stated that 2D models provide results qualitatively comparable with those obtained using $3 \mathrm{D}$ models in a wide range of frequencies.

In the light of this, the 2D model has been considered appropriate to output results consistent with the aim of the present study.

4.1. Material Constitutive Model and Type of FEM Elements Applied. Linear viscoelastic constitutive model for the embankment and the soil medium materials has been adopted to carry out the investigation, according to some previous studies $[20,21]$. Both the embankment and the soil medium have been meshed with triangular elements having 6 nodes. The mechanical parameters assigned to each element are the Young modulus E, Poisson's ratio $v$, and the mass density $\rho$, while the damping behaviour has been introduced by means of the Rayleigh coefficients $\alpha$ and $\beta$. The damping matrix [C] has been defined on the basis of the quoted Rayleigh coefficients $\alpha$ and $\beta$.

The presence in the analysed scheme of a rigid base has been taking into account by introducing in the model fixities at bedrock.

4.2. Modelling of the Rail Source. The railway source has been modelled applying in the surface of the embankment below the ballast a stress having a value equal to the dynamic stress produced in the contact surface ballast-embankment.

In particular, according to [6], two concentrated linear loads acting over the rails have been considered. The time history of each load consists of four consecutive impulses; each impulse have a time duration of 0.02 seconds and an amplitude equal to $1000 \mathrm{KN}$. Since only the embankment has been modelled, the dynamic pressure produced by the linear loads in the contact surface ballast/embankment, having dimensions of about $2,5 \mathrm{~m} \times 1 \mathrm{~m}$, has been fixed in $400 \mathrm{kN} / \mathrm{m}^{2}$ (Figure 3 ) while its time history is the one plotted in Figure 4. 
TABLE 3: Mechanical characteristics of the soil medium.

\begin{tabular}{lcccccc}
\hline Type of soil & $G\left(\mathrm{MN} / \mathrm{m}^{2}\right)$ & $\rho\left(\mathrm{N} / \mathrm{m}^{4} \mathrm{sec}^{2}\right)$ & $v$ & $\xi$ & $V_{s}(\mathrm{~m} / \mathrm{sec})$ & $V_{r}(\mathrm{~m} / \mathrm{sec})$ \\
\hline Clay & 20.7 & 1583.8 & 0.3 & 0.06 & 114.3 & 108.1 \\
Sand & 138 & 1682.8 & 0.3 & 0.04 & 286.3 & 270.9 \\
\hline
\end{tabular}

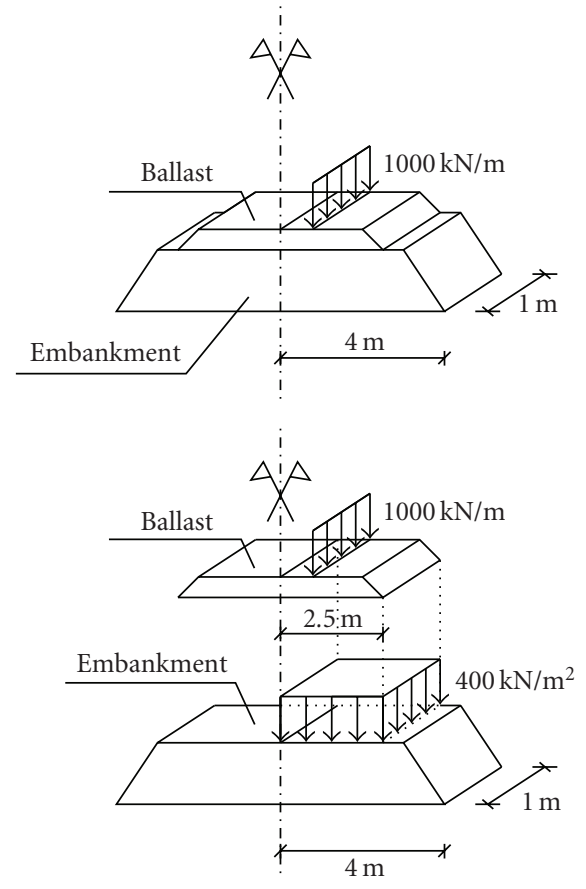

FIgURE 3: Loading conditions.

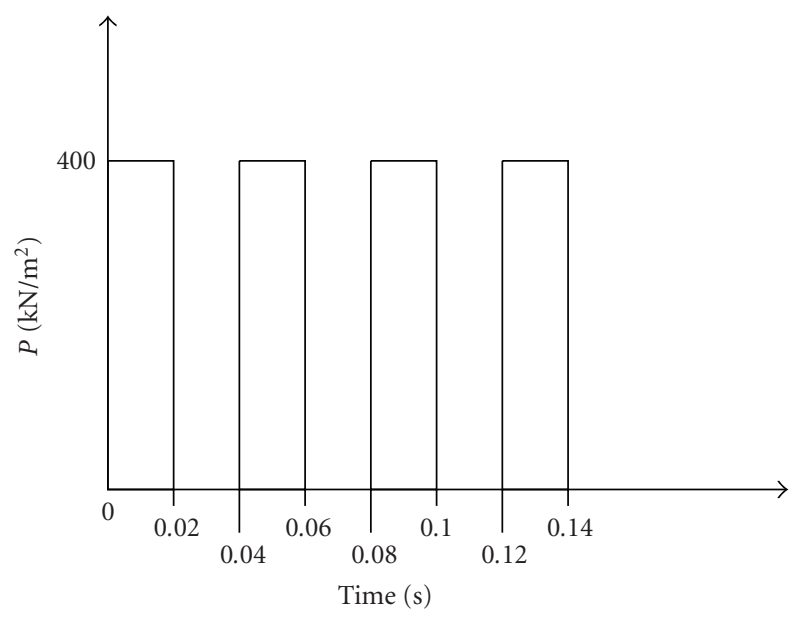

FIgUre 4: Time history of the rail force.

The applied load function gives a significant contribution for the frequencies included in the range from 20 to $35 \mathrm{~Hz}$, with a central frequency of about $27 \mathrm{~Hz}$. This range, in the present study-case, is representative of the frequencies produced by the passage of a freight train at the velocity of $60 \mathrm{~km} / \mathrm{h}$, and having a boogie axle distance of $9 \mathrm{~m}$. The load can appear overabundant but it takes into account the dynamic amplification factor by the shortest wavelength $[6,22]$.

4.3. Finite Element Mesh. The definition of the model mesh is in general a compromise between the necessity to restrict the number of the elements and that to limit their dimensions. However, the element's dimension must be accurately chosen in order to adequately represent the deformed shape associated to the wave lengths $\lambda$ at the different harmonic frequencies $f$ imputed.

If $f_{\max }$ is the greatest frequency of the significant components of the Fourier spectrum and $V_{j}$ is the propagation speed of the waves ( $P$-waves, $S$-waves, or $R$-waves) in the material, the dimension of the mesh element for the material should satisfy the following relation:

$$
d \leq \frac{\lambda_{\min }}{k}=\frac{V_{j}}{k \cdot f_{\max }},
$$

where $k$ is a coefficient ranging from 4 to 10 according to the type of finite element and to its shape function [23].

In the light of this, the maximum dimension of the element has been defined in the following way:

(i) on the basis of the material properties, there has been calculated the minimum wave propagation velocity for the considered domain; that is, for the superficial elements there has been considered the Rayleigh's velocity $V_{r}$, for the elements close to deep source the shear velocity $V_{s}$;

(ii) there has been defined the maximum value of the appropriate frequency $f_{\max }$; that is, in this study, according to the railway source model, $f_{\max }$ has been set up equal to $35 \mathrm{~Hz}$;

(iii) there has been calculated, using the expression (1), the minimal dimension of the element.

By applying this procedure to the two types of soil medium, there has been obtained

Clay $\Rightarrow V_{r}=108.16 \mathrm{~m} / \mathrm{sec} ; f_{\max }=35 \mathrm{~Hz} ; k=6 \Rightarrow$ $d_{A} \leq 0.51 \mathrm{~m}$

Sand $\Rightarrow V_{r}=270.94 \mathrm{~m} / \mathrm{sec} ; f_{\max }=35 \mathrm{~Hz} ; k=6 \Rightarrow d_{S} \leq$ $1.29 \mathrm{~m}$.

By considering these dimensions, the mesh has been made as follows:

(i) in the part of the model where the response is significant to the aim of the study, corresponding to a strip, large about $40 \mathrm{~m}$ and $40 \mathrm{~m}$ deep, closest to the rail track, a maximum dimension of $0.5 \mathrm{~m}$ has been assigned to the elements; this mesh has been designed to be denser in this focal area in order to achieve a good precision; 


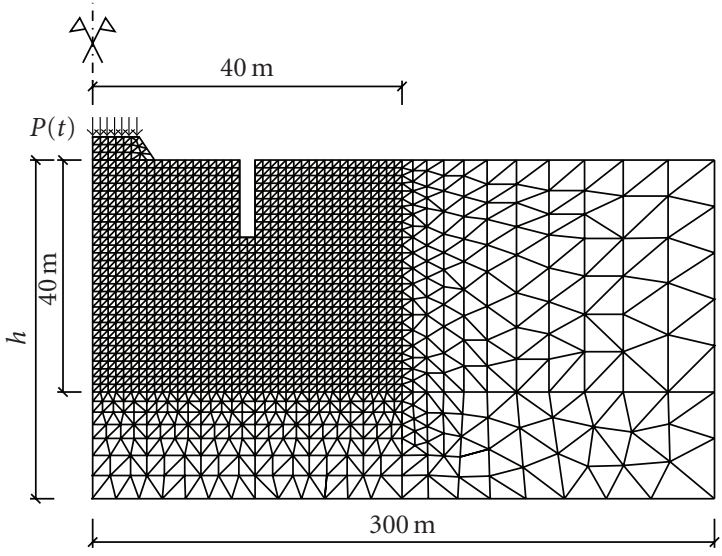

Figure 5: Scheme of FEM mesh.

(ii) in the part of the model included between the quoted part and the lower edge a maximum dimension of $0.5 \mathrm{~m}$ has been assigned when the clayey soil has been analysed, while in the case of sandy soil the dimensions of the element gradually increase until $1.5 \mathrm{~m}$ near the lower edge;

(iii) externally to the above mentioned areas, the dimensions of the elements increase gradually reaching the value of $3 \mathrm{~m}$ in proximity of the vertical boundary far $300 \mathrm{~m}$ from the symmetry axis.

The linear variation of the mesh dimensions, from the side $y=40 \mathrm{~m}$ to the boundary, has been chosen in accordance with other similar works $[24,25]$.

The scheme of the finite element mesh is shown in Figure 5.

It can be observed that outside the zone of interest $(40 \mathrm{~m} \times 40 \mathrm{~m})$, the growth of the dimensions of the element in connection with the time step does not produce effects in the part of the model where is sited the trench; in fact, the vibratory waves, also when undergo reflections in correspondence of the vertical boundary right hand, cannot reach this zone before the end of the analysis.

4.4. Courant's Condition. The time step integration has been assigned taking into account the Courant condition, that defines the maximum time step as [26]

$$
\frac{V_{j} \cdot \Delta t}{h}=C, \quad C \leq 1
$$

in which $V_{j}$ is the velocity of the considered wave, $h$ is the maximum spatial displacement admitted for the perturbation that in FEM models is generally assumed equal to the dimension of the mesh, and $C$ is the Courant number.

It should be noted that in the problems involving different type of waves, like the propagation of the $P$ and $S$ waves in the elastic space, the time step is imposed by the greatest propagation velocity, while the element's dimensions depend on the lowest velocity. So, a great difference between the greatest and the lowest propagation velocity requires a small finite element and a short-time step. that

Having in mind this, in this task there has been assumed

Clay $\Rightarrow V_{p}=191.55 \mathrm{~m} / \mathrm{sec}, d_{A, \min }=0.5 \mathrm{~m} \Rightarrow \Delta t \leq$ $0.0026 \mathrm{sec}$; there has been chosen $\Delta t=0.002 \mathrm{sec}$.

Sand $\Rightarrow V_{p}=479.83 \mathrm{~m} / \mathrm{sec}, d_{S, \min }=0.5 \mathrm{~m} \Rightarrow \Delta t \leq$ $0.00104 \mathrm{sec}$; there has been chosen $\Delta t=0.001 \mathrm{sec}$.

In both cases of clay and sand the FEM simulations have been performed in a time of $T=0.75 \mathrm{sec}$; this time is sufficient to permit the complete passage of the dynamic perturbation in the examined zone.

4.5. Boundary Condition. Boundary conditions have been imposed in the model in consideration of the geometric and mechanical conditions of symmetry and taking into account the need to limit the errors, namely, the difference existing between the theoretical value of the stress in a point of the boundary and the value obtained in presence of the restraints.

FE calculations need a self-consistent simulation area to operate on. Especially in the case of wave propagation, special boundary conditions have to be incorporated into the calculation scheme [27]. Many authors have proposed special absorbing boundary conditions (ABCs) for the FE calculation of elastic waves. In these works the boundary conditions suggested from Lysmer and Kuhlemeyer have been applied [28]:

$$
\begin{gathered}
\sigma=-\rho c_{d} V_{n}, \\
\tau=-\rho c_{s} V_{t},
\end{gathered}
$$

where $\sigma$ and $\tau$ are the normal and the shear stress, $\rho$ the material density, and $c_{d}$ and $c_{s}$ the longitudinal and the shear wave velocities of the transmitting media. These equations reveal that the magnitude of these stresses at the boundaries is proportional to the particle velocities in the normal $\left(V_{n}\right)$ and in the tangential $\left(V_{t}\right)$ directions. Lysmer's dampers placed on the artificial boundary are effective in reducing unwanted wave reflections if the boundary of the finite element mesh is sufficiently far outward.

In the light of this, there has been applied

(i) in the symmetry edge, symmetry restraints like bipendulum in $y$ direction;

(ii) in the lower edge, fixities at the bedrock;

(iii) in the right-side edge, Lysmer's dampers in the directions $y$ and $z$.

The right-side edges have been applied at a distance of $300 \mathrm{~m}$ from the source; the errors obtained in the boundary have been

(i) $\varepsilon\left(\sigma_{n}\right)=0.10 \%$ and $\varepsilon(\tau)=0.45 \%$, in the case of the clayey soil medium;

(ii) $\varepsilon\left(\sigma_{n}\right)=0.26 \%$ and $\varepsilon(\tau)=1.12 \%$, in the case of the sandy soil medium.

4.6. The Determination of the Rayleigh Damping Coefficients $\alpha$ and $\beta$. In an FEM model the damping behaviour can be 


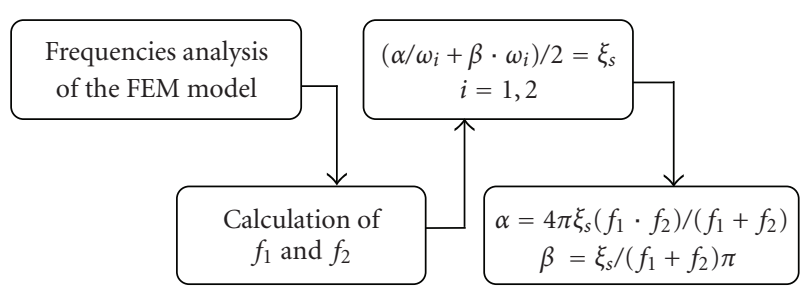

FIGURE 6: Rayleigh's parameters determination.

modelled using Rayleigh's method. The damping matrix can be obtained from the following relation:

$$
[C]=\alpha[M]+\beta[K],
$$

where $[M]$ and $[K]$ are, respectively, the mass matrix and the stiffness matrix.

The $\alpha$ and $\beta$ damping coefficients [23, 29] have been determined following the procedure described below and shown in Figure 4.

(i) For each 2D model, a frequency analysis has been carried out and the first resonance frequency $f_{1}$ has been calculated.

(ii) There has been calculated the frequency $f_{2}$ by means of the relation $f_{2}=n f_{1}$, where $n$ is the first odd number greater than the ratio $f_{i} / f_{1}$, being $f_{i}$ the dominant frequency. In this case $f_{i}$ has been fixed equal to $27 \mathrm{~Hz}$, like the medium frequency of the load function.

(iii) Rayleigh's parameters value has been calculated by establishing that, for frequencies equal to $f_{1}$ and $f_{2}$, the damping coefficient of the element must be equal to the value $\xi_{s}$, which is proper to the examined soil. In this way there has been introduced a subdamping for the frequencies ranging from $f_{1}$ to $f_{2}$ and a superdamping for the other frequencies not included in this range.

For the clayey soil Rayleigh's parameters have been calculated assuming that a damping ratio $\xi_{s}$ is equal to 0.06 , while for the sandy one there has been assumed that $\xi_{s}$ is equal to 0.04 .

In Figures 7 and 8, we can see examples of damping diagrams for clayey and sandy soil medium.

4.7. Validation Model. In order to validate the model, results by Beskos et al. [11] have been considered and used. Results concern an open trench having a depth of $2.8 \mathrm{~m}\left(1 \lambda_{r}\right)$ and a width of $0.28 \mathrm{~m}\left(0.1 \lambda_{r}\right)$; it is made in an elastic half-space at a distance of $10.04 \mathrm{~m}\left(5 \lambda_{r}\right)$ from the axis of a rigid foundation having a width of $0.7 \mathrm{~m}$ and subjected to a harmonic load with frequency of $50 \mathrm{~Hz}$.

In particular the comparison between the results given by the proposed 2D FEM model and the Beskos one has been performed considering the trend of the attenuation ratio $A_{r}$ versus the normalized distance $y / \lambda_{r}$, where $\lambda_{r}$ is the length of Rayleigh's waves generated from the source and $y$ the horizontal distance of the generic point from the axis of the source.

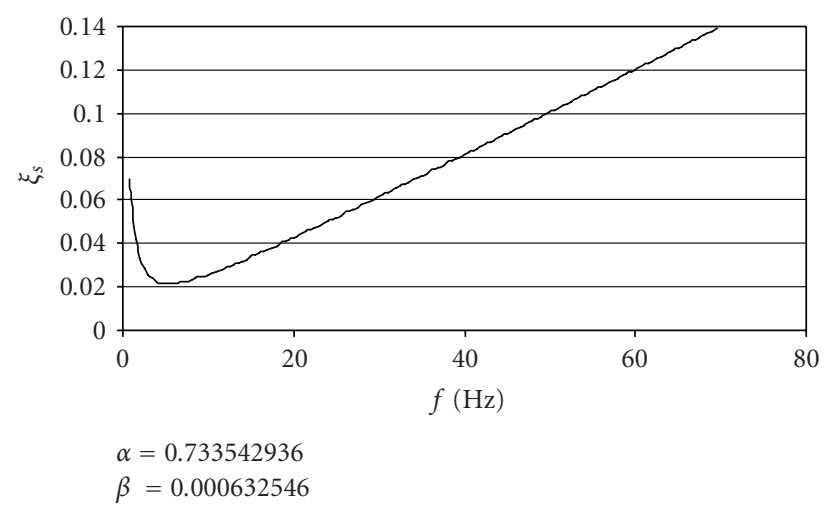

Figure 7: Damping ratio $\xi_{s}$ versus frequency for clay bank of $25 \mathrm{~m}$ depth.

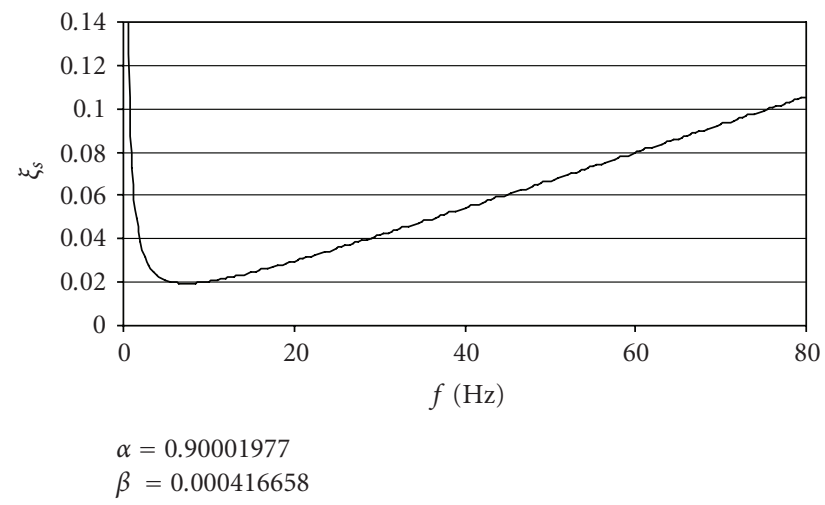

FIGURE 8: Damping ratio $\xi_{s}$ versus frequency for sand bank of $40 \mathrm{~m}$ depth.

In each point of both vibration-sensitive site and site close to the trench the attenuation ratio $A_{r}$ has been defined as the ratio between the maximum value of the kinematic parameters ( $y$ and $z$ displacements and $y$ and $z$ velocities) in presence of trench and the same value without trench:

$$
\begin{gathered}
A_{r}=(\text { max value of the kinematic parameters in a point } \\
\text { with trench }) /
\end{gathered}
$$

(max value of the kinematic parameters in a point without trench).

The attenuation ratio has been calculated not only for the vertical displacement (dir. $z$ ), as it is usual in literature, but also for the horizontal displacement $(\operatorname{dir} y)$ and for the horizontal and vertical velocities.

Since the sites are not points but large zones and considering the evolution of the attenuation ratio increasing distance (Figure 7), the effectiveness of the trench in these sites has been evaluated in terms of average attenuation ratio:

$$
A_{m}=\frac{1}{C} \int_{0}^{c} A_{r}(y) d y,
$$




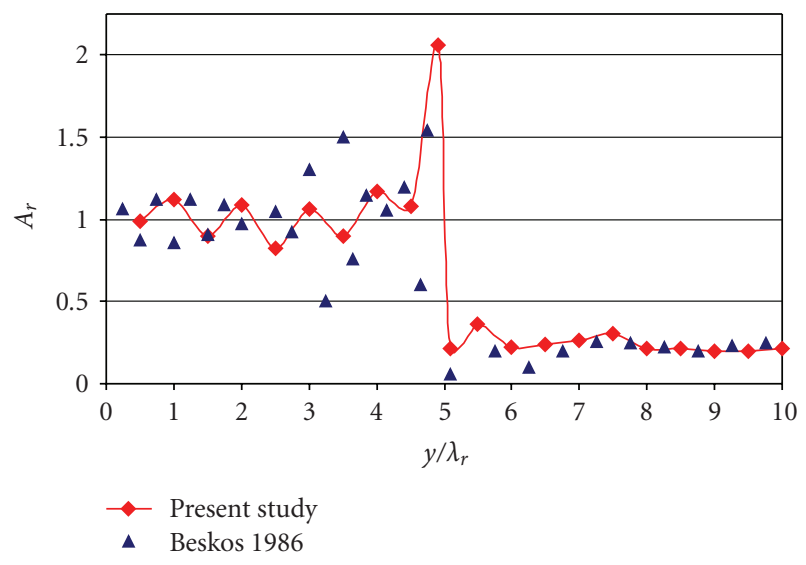

Figure 9: Model results versus Beskos results. where $C$ is the length of the investigated zone (equal to $10 \mathrm{~m}$ for vibration sensitive site and $5 \mathrm{~m}$ for site close to the trench).

As it can see in Figure 9, a good agreement between the numerical results from Beskos simulation and the ones obtained with the proposed model has been achieved. The model has given good results also in others experimental applications [29] and thus it has been applied in the present study.

4.8. Results. From the numerical FEM simulations of the 540 scenarios the values of the displacements and velocities have been obtained; the minimum and the maximum values reached in the significant points are summarised in Table 4 .

In Figure 10 two samples of time history of displacement and velocity in a point are plotted.

When the horizontal propagation of in-plane waves is considered, the Rayleigh waves and the $P$-waves are the main causes of the ground motion.

At quite distance $\left(5 \lambda_{r}\right)$ from a vibratory source the ground motion is due to the Rayleigh waves energy.

If an open trench is excavated near the source, the incidence of the waves on this obstacle (discontinuity field) gives rise to reflected and transmitted body waves. Behind the open trench, as the distance increases, the transmitted $P$ waves get partially into Rayleigh waves (phenomenon known as mode conversion). Therefore, the Rayleigh waves are the main cause of the ground motion [13].

According to this phenomenon the results show a great attenuation of displacement and velocity passing from the site close to the trench to the vibration sensitive site (see Table 4).

To evaluate the effectiveness of the trench there has been adopted the very strict following criterion: a trench is effective in screening the ground borne vibration if the value of the attenuation ratio is lower than 0.4 for every considered displacement and velocity.

The analysis of the FEM results for the two types of ground has been performed having in mind this criterion.

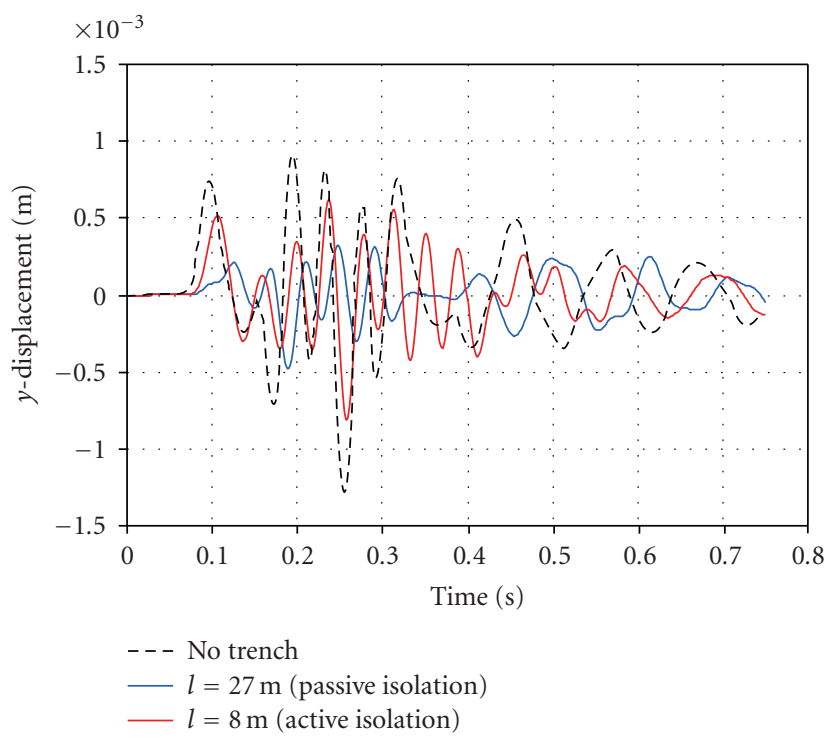

(a)

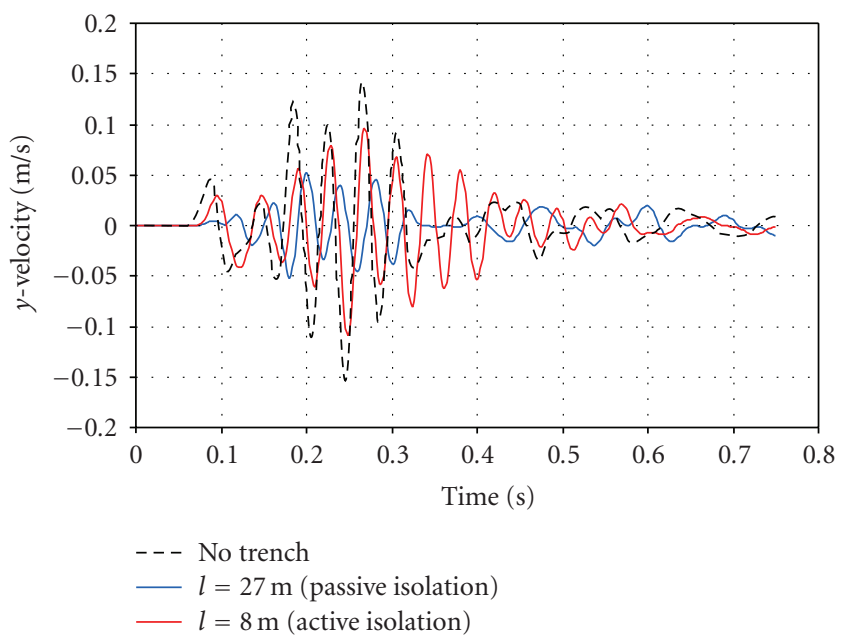

(b)

FigURE 10: Samples of results in a point in case of sandy soil.

The results give some indications for the design of the open trenches in both cases of site close to the trench and vibration-sensitive site (see Tables 5 and 6 ).

The effect of frequency is fully taken into account by normalizing the open trench dimensions with respect to the Rayleigh wavelength.

The obtained results are very strict in regard to the dimensions and the position of the trench because these features depend on the ratio $d / h$ and so on the finite value of the layer thickness.

For instance, in clayey soil results show that the trench is effective if it is $5 \mathrm{~m}$ deep and far away at least $12 \mathrm{~m}$ from the source, for thickness layer equal to $10 \mathrm{~m}$ or $2 \mathrm{~m}$ deep and far away at least $24 \mathrm{~m}$ from source, and for layer thickness equal to $3 \mathrm{~m}$.

Some results of the parametric studies carried out are plotted in Figure 11. It can be seen that the efficiency of 


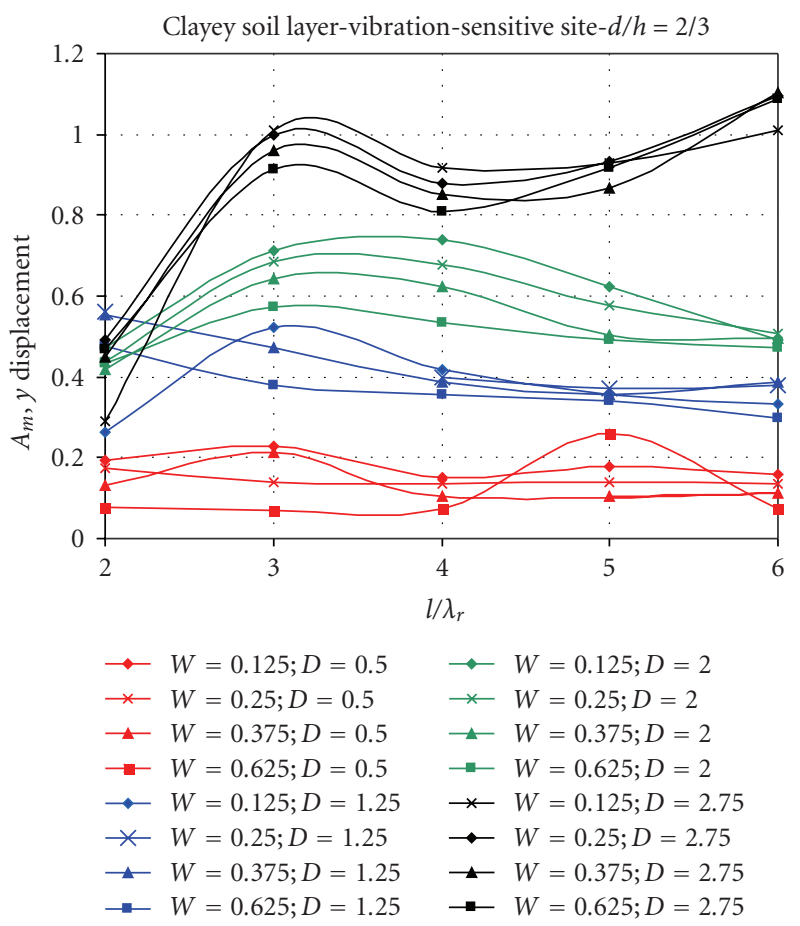

(a)

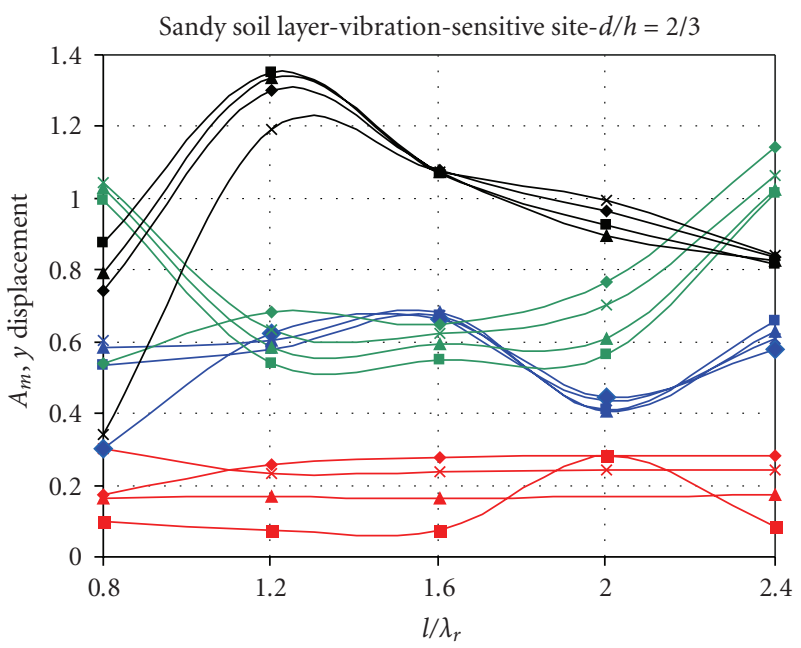

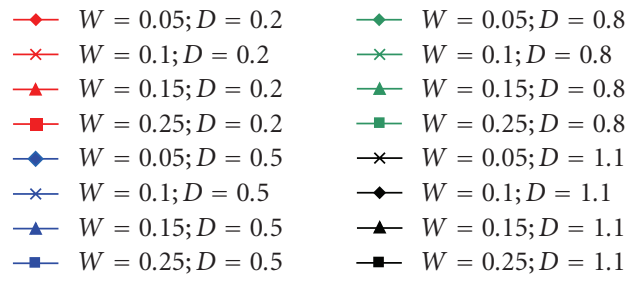

(b)

FIgURE 11: Some results of parametric study $\left(W=w / \lambda_{r}, D=d / \lambda_{r}\right)$.

TABLE 4: Maximum and minimum values of displacements and velocities.

\begin{tabular}{|c|c|c|c|c|}
\hline \multicolumn{5}{|c|}{ Site close to the trench } \\
\hline Soil type & $\begin{array}{l}\text { Velocity } \\
\text { dir. } y \text { min } \\
\max \\
(\mathrm{m} / \mathrm{sec})\end{array}$ & $\begin{array}{l}\text { Velocity } \\
\text { dir. } z \text { min } \\
\max \\
(\mathrm{m} / \mathrm{sec})\end{array}$ & $\begin{array}{l}\text { Displacement } \\
\text { dir. } y \text { min } \\
\max (m)\end{array}$ & $\begin{array}{l}\text { Displacement } \\
\text { dir. } z \text { min } \\
\max (\mathrm{m})\end{array}$ \\
\hline \multirow{2}{*}{ Sand } & 0.13 & 0.09 & 0.00098 & 0.00056 \\
\hline & 0.35 & 0.25 & 0.00252 & 0.00619 \\
\hline \multirow{2}{*}{ Clay } & 0.16 & 0.13 & 0.00564 & 0.00369 \\
\hline & 0.29 & 0.48 & 0.01084 & 0.02509 \\
\hline \multicolumn{5}{|c|}{ Site vibration-sensitive } \\
\hline Soil type & $\begin{array}{l}\text { Velocity } \\
\text { dir. } y \text { min } \\
\max \\
(\mathrm{m} / \mathrm{sec})\end{array}$ & $\begin{array}{l}\text { Velocity } \\
\text { dir. } z \text { min } \\
\max \\
(\mathrm{m} / \mathrm{sec})\end{array}$ & $\begin{array}{l}\text { Displacement } \\
\text { dir. } y \text { min } \\
\max (\mathrm{m})\end{array}$ & $\begin{array}{l}\text { Displacement } \\
\text { dir. } z \text { min } \\
\max (\mathrm{m})\end{array}$ \\
\hline \multirow{2}{*}{ Sand } & 0.052 & 0.035 & 0.00078 & 0.00009 \\
\hline & 0.21 & 0.20 & 0.00198 & 0.00341 \\
\hline \multirow{2}{*}{ Clay } & 0.035 & 0.028 & 0.00177 & 0.00040 \\
\hline & 0.27 & 0.30 & 0.01084 & 0.01714 \\
\hline
\end{tabular}

TABLE 5: Design parameters for trench—site close to the trench.

\begin{tabular}{|c|c|c|c|c|c|c|}
\hline \multirow{2}{*}{$d / h$} & \multicolumn{3}{|c|}{ Clayey soil layer $\left[\lambda_{r}=4,00 \mathrm{~m}\right]$} & \multicolumn{3}{|c|}{ Sandy soil layer $\left[\lambda_{r}=10,00 \mathrm{~m}\right]$} \\
\hline & $d$ & $l$ & $w$ & $d$ & $l$ & $w$ \\
\hline $1 / 5$ & - & - & - & $0.2 \lambda_{r}$ & $2 \lambda_{r}$ & $\leq 0.1 \lambda_{r}$ \\
\hline $1 / 2$ & $1.25 \lambda_{r}$ & $>3 \lambda_{r}$ & all & $0.5 \lambda_{r}$ & $\geq 1.2 \lambda_{r}$ & all \\
\hline $2 / 3$ & $<2.75 \lambda_{r}$ & $6 \lambda_{r}$ & all & $0.2 \lambda_{r}$ & all & all \\
\hline
\end{tabular}


TABLE 6: Design parameters for trench—vibration-sensitive site.

\begin{tabular}{ccccccc}
\hline$d / h$ & Clayey soil layer $\left[\lambda_{r}=4,00 \mathrm{~m}\right]$ & & \multicolumn{3}{c}{ Sandy soil layer $\left[\lambda_{r}=10,00 \mathrm{~m}\right]$} \\
& $d$ & $l$ & $w$ & $d$ & $l$ & $w$ \\
$1 / 5$ & - & - & - & $0.2 \lambda_{r}$ & $0.8 \lambda_{r}$ & $0.05 \lambda_{r}$ \\
$1 / 2$ & $1.25 \lambda_{r}$ & $>3 \lambda_{r}$ & all & $0.5 \lambda_{r}$ & $0.8 \lambda_{r}$ & $0.05 \lambda_{r}$ \\
$2 / 3$ & $0.5 \lambda_{r}$ & all & all & $0.2 \lambda_{r}$ & $\geq 0.8 \lambda_{r}$ & all \\
\hline
\end{tabular}

TABLE 7: Percentage of contribution to the attenuation of the displacements-site close to the trench.

\begin{tabular}{lccc}
\hline \multicolumn{2}{c}{$\begin{array}{c}\text { Attenuation ratio for horizontal displacements } \\
\text { Percentage of contribution }\end{array}$} & \multicolumn{2}{c}{$\begin{array}{c}\text { Attenuation ratio for vertical displacements } \\
\text { Parameters }\end{array}$} \\
\hline$d$ & $28.00 \%$ & $d$ & $31.00 \%$ \\
$d / h$ & $26.00 \%$ & $d / h$ & $28.00 \%$ \\
$V_{s}$ & $17.00 \%$ & $l$ & $14.50 \%$ \\
$\xi$ & $15.50 \%$ & $V_{s}$ & $13.00 \%$ \\
$l$ & $10.50 \%$ & $\xi$ & $9.00 \%$ \\
$w$ & $3.00 \%$ & $w$ & $4.50 \%$ \\
\hline
\end{tabular}

TABLE 8: Percentage of contribution to the attenuation of the velocities-site close to the trench.

\begin{tabular}{lccc}
\hline & $\begin{array}{c}\text { Attenuation ratio for horizontal velocities } \\
\text { Percentage of contribution }\end{array}$ & \multicolumn{2}{c}{$\begin{array}{c}\text { Attenuation ratio for vertical velocities } \\
\text { Percentage of contribution }\end{array}$} \\
\hline$d$ & $31.00 \%$ & $d$ & $27.00 \%$ \\
$d / h$ & $23.00 \%$ & $d / h$ & $26.00 \%$ \\
$\xi$ & $17.00 \%$ & $l$ & $15.00 \%$ \\
$l$ & $14.00 \%$ & $V_{s}$ & $13.50 \%$ \\
$V_{s}$ & $11.00 \%$ & $\xi$ & $11.50 \%$ \\
$w$ & $4.00 \%$ & $w$ & $7.00 \%$ \\
\hline
\end{tabular}

TABLE 9: Percentage of contribution to the attenuation of the displacements-vibration-sensitive site.

\begin{tabular}{lccc}
\hline \multicolumn{2}{c}{$\begin{array}{c}\text { Attenuation ratio for horizontal displacements } \\
\text { Percentage of contribution }\end{array}$} & \multicolumn{2}{c}{$\begin{array}{c}\text { Attenuation ratio for vertical displacements } \\
\text { Parameters }\end{array}$} \\
\hline$d$ & $36.00 \%$ & $d$ & $42.00 \%$ \\
$d / h$ & $30.00 \%$ & $d / h$ & $26.00 \%$ \\
$V_{s}$ & $13.00 \%$ & $V_{s}$ & $12.00 \%$ \\
$\xi$ & $11.00 \%$ & $\xi$ & $11.00 \%$ \\
$l$ & $7.00 \%$ & $l$ & $7.00 \%$ \\
$w$ & $3.00 \%$ & $w$ & $2.00 \%$ \\
\hline
\end{tabular}

TABLE 10: Percentage of contribution to the attenuation of the velocities—vibration-sensitive site.

\begin{tabular}{|c|c|c|c|}
\hline \multicolumn{2}{|c|}{ Attenuation ratio for horizontal velocities } & \multicolumn{2}{|c|}{ Attenuation ratio for vertical velocities } \\
\hline Parameters & Percentage of contribution & Parameters & Percentage of contribution \\
\hline$d$ & $34.00 \%$ & $d$ & $35.00 \%$ \\
\hline$d / h$ & $27.00 \%$ & $d / h$ & $22.50 \%$ \\
\hline$V_{s}$ & $17.00 \%$ & $V_{s}$ & $16.00 \%$ \\
\hline$\xi$ & $14.00 \%$ & $\xi$ & $15.50 \%$ \\
\hline$l$ & $5.00 \%$ & $l$ & $8.00 \%$ \\
\hline$w$ & $3.00 \%$ & $w$ & $3.00 \%$ \\
\hline
\end{tabular}


the trench is in general independent from the width of the trench; therefore it can be established according to technicaleconomic criteria, while the depth affects significantly the attenuation process.

\section{ANNs Analysis}

5.1. Introduction. Artificial neural networks (ANNs) are a tool that simulates the biological processes and, as demonstrated by the wide applications in engineering field, they are able to solve functional mapping problems. ANNs are an assemblage of mathematical simple computational elements called neurons.

In particular the back propagation neural network (BPNN) is a collection of neurons distributed over an input layer, that contain the input variables of the problem, one or more hidden layers with a certain number of nodes, and an output layer with a number of nodes equal to the output variables. The nodes between layers are connected by the links having a weight that describes quantitatively the strength of the connection.

A significant effort is required in the selection of the ANN architecture, particularly, as it is obvious, in the definition of the hidden layers and the corresponding nodes.

The learning process utilized for this type of ANN is the "back-propagation learning" which consists in an error minimization technique [30]. Inputs from the mapping examples are propagated through each layer and give the outputs that are compared with the correct answers; the difference represents the error. This error is propagated backward through the network and the connection weights are individually adjusted so as to reduce and minimize the error.

To ensure an efficient convergence and the desired performance of the trained network, several parameters are incorporated in the training phase. These parameters include the learning rate, the momentum term, and the number of training iterations. The learning rate is a factor that proportions the amount of the adjustment applied at each time the weight is updated. The use of a momentum term could carry the weight change process through one or more local minima and get it into global minima. The early stopping method and the number of training epochs determine the training stop criteria [31].

5.2. Neural Network Application. For the present application the neural networks have been trained and tested with the data given from the FEM modelling of the 540 scenarios.

In details, the input data chosen for the neural analysis have been 6 and precisely: width of the trench $(w)$, depth of the trench $(d)$, ratio of depth of the trench to thickness of the soil layer $(d / h)$, shear wave velocity $\left(V_{s}\right)$, and damping ratio $(\xi)$, while the output data have been the average attenuation ratio of the horizontal and vertical displacements, and the average attenuation ratio of the horizontal and the vertical velocity. In fact, the neural network application aimed at evaluating the contribution of each input factor in the attenuation of the displacements and velocities.
To this purpose a great number of trial ANNs with one, two, and three hidden layer have been trained to evaluate the performance of different network architectures in the comprehension and generalisation of the problem.

The training procedure has been tested using a test set of examples having a percentage of $10 \%$ of the training set; the prediction performance of the developed model has been exhibited in the test set.

The error term, represented by the mean square error (RMS error), computed for the validation input-output pairs, has been monitored during the training process of the networks. The error normally decreases during the initial phase of training. However, when the network begins to overfit the data (a situation arising when ANN works well only with the training data), the error on the validation set will typically begin to increase. When the validation error increased for a specified number of iterations, training was stopped and the weights at the minimum of the validation error were saved. This point is the point of maximum generalization [30].

Considering this, networks with errors comprising in the following ranges have been selected: RMS error training set: $0.02-0.1$; RMS error test set: 0.02-0.12; Correlation training set: $0.5-1$. It has been stated that the neural networks having the training parameters included in the quoted ranges provide acceptable results.

The analysis of the training and the testing phases of these neural networks allowed to evaluate the percentage of contribution of each input factor in the output determination. In other terms the study allowed to assess qualitatively and quantitatively the influence of the features of the trench and the soil medium in the attenuation process of ground-borne vibrations.

5.3. Results. The results obtained for both vibration-sensitive site and site close to the trench are explained in the following.

5.3.1. Site Close to the Trench. Regarding the attenuation ratio of the horizontal and vertical displacements, the neural network analysis has given the percentages of contribution summarised in Tables 7 and 8.

By analysing the results it can be deduced that for both vertical and horizontal displacements the percentage of contribution to the attenuation ratio of the depth of the trench $d$ and of the ratio $d / h$ is close to the $60 \%$. The influence of the terrain, expressed by $V_{s}$ and $\xi$, ranges from $32 \%$ for horizontal displacements to about $22 \%$ for vertical displacements. The influence of the distance of the trench from rail $l$ is evaluated around the $10 \%-14 \%$ while the width of trench $w$ has a marginal weight of about 3-5\%.

Regarding the attenuation of the velocities the hierarchy of influence of the parameters seems to be the same of the displacements: the influence of the depth of the trench $d$ and of the ratio $d / h$ is preponderant (about $50 \%$ ), while the influence of the terrain is evaluated around the 25\%-28\% while, in contrast to the results found for the displacements, there appears significant the distance of the trench from rail $l$ that contributes for around the $15 \%$ to the attenuation 
process. The width of the trench $w$, even if with the different weights for horizontal (4\%) and vertical (7\%) velocities, gives a poor contribution to the attenuation phenomenon.

5.3.2. Vibration-Sensitive Site. The percentages of contribution to the attenuation ratio of the horizontal and vertical displacements are summarised in Tables 9 and 10 .

In this site the influence of the depth of the trench $d$ on the attenuation ratio of the vertical and horizontal displacements is very significant $(36 \%-42 \%)$ and together with the ratio $d / h$ seems to govern preponderantly the attenuation process. The other factors $\left(V_{s}, \xi, l\right)$ have an influence comparable (each ranged from 7 to $13 \%$ ) and lesser than $d$ and $d / h$. Once again the width $w$ of the trench is not much significant.

Regarding the attenuation ratio of the horizontal and vertical velocities the trend is the same of the displacements but the contribution of the terrain's parameters ( $V_{s}$ and $\xi$ ) is higher (about 30\%). The distance of the vibration sensitive site from the source $l$ and even more the width $w$ of the trench have a low contribution.

\section{Conclusions}

In order to intercept the elastic waves and in particular Rayleigh's ones generated by the moving source from reaching buildings between the railways and the sensitive site, vibration screening may be established by means of the open trenches. Several factors determine the effectiveness of the open trenches in the screening of the ground borne vibrations in soil medium.

In the present work, as outcomes of an FEM modelling of the problem and of an analysis carried out by means of the neural network, the weights of the main geometric features of both the trench and the soil medium have been established.

The main conclusions of this application are the following:

(i) first of all, the screening performance of the trench is mainly affected by the depth of the trench, in both cases of site close to the trench and vibrationsensitive site. From ANN analysis the percentage of contribution of this geometric parameter in reducing displacements and velocities of vibration reached the $25 \%-35 \%$;

(ii) layer thickness increasing, the attenuation ratio increases according to the depth of the trench; to this reason, it appears very noteworthy in the topic not only the depth $d$ of the trench but also the ratio $d / h$ (depth of the trench/ thickness of the bank); the two parameters $d$ and $d / h$ govern the attenuation process with a percentage of contribution more than $50 \%$;

(iii) to the aim of evaluating the effectiveness of the trench we have to analyse all the kinematic parameters and not only the displacement in one direction, generally the direction $z$; in fact, some geometric configura- tions able to reduce one kinematic parameters can be inefficient with regard to the attenuation of the other kinematic parameters.

\section{References}

[1] H.-H. Hung and Y.-B. Yang, "A review of researches on ground-borne vibrations with emphasis on those induced by trains," Proceedings of the National Science Council, Republic of China, Part A, vol. 25, no. 1, pp. 1-16, 2001.

[2] Y.-B. Yang and H.-H. Hung, "A parametric study of wave barriers for reduction of train-induced vibrations," International Journal for Numerical Methods in Engineering, vol. 40, no. 20, pp. 3729-3747, 1997.

[3] R. D. Woods, "Screening of surface waves in soils," Journal of the Soil Mechanics and Foundations Division, vol. 94, no. 4, pp. 951-979, 1968.

[4] D. D. Barkam, Dynamics of Bases and Foundations, McGrawHill, New York, NY, USA, 1962.

[5] R. K. Shrivastava and N. S. V. Kameswara Rao, "Response of soil media due to impulse loads and isolation using trenches," Soil Dynamics and Earthquake Engineering, vol. 22, no. 8, pp. 695-702, 2002.

[6] M. Adam and O. von Estorff, "Reduction of train-induced building vibrations by using open and filled trenches," Computers and Structures, vol. 83, no. 1, pp. 11-24, 2005.

[7] A. Karlström and A. Boström, "Efficiency of trenches along railways for trains moving at sub- or supersonic speeds," Soil Dynamics and Earthquake Engineering, vol. 27, no. 7, pp. 625641, 2007.

[8] L. Andersen and S. R. K. Nielsen, "Reduction of ground vibration by means of barriers or soil improvement along a railway track," Soil Dynamics and Earthquake Engineering, vol. 25, no. 7-10, pp. 701-716, 2005.

[9] H. J. Dolling, "Die Abschirmung von Erschütterungen durch Bodenschlitze," Bautechnik, vol. 6, pp. 193-204, 1970.

[10] W. A. Haupt, "Model tests on screening of surface waves," in Proceedings of the 10th International Conference on Soil Mechanics and Foundation Engineering, vol. 3, pp. 215-222, Stockholm, Sweden, June 1981.

[11] D. E. Beskos, B. Dasgupta, and I. G. Vardoulakis, "Vibration isolation using open or filled trenches-part 1: 2-D homogeneous soil," Computational Mechanics, vol. 1, no. 1, pp. 43-63, 1986.

[12] B. Dasgupta, D. E. Beskos, and I. G. Vardoulakis, "Vibration isolation using open or filled trenches-part 2: 3-D homogenous soil," Computational Mechanics, vol. 6, pp. 129-142, 1990.

[13] S. Ahmad and T. M. Al-Hussaini, "Simplified design for vibration screening by open and in-filled trenches," Journal of Geotechnical Engineering, vol. 117, no. 1, pp. 67-88, 1991.

[14] T. M. Al-Hussaini and S. Ahmad, "Design of wave barriers for reduction of horizontal ground vibration," Journal of Geotechnical Engineering, vol. 117, no. 4, pp. 616-636, 1991.

[15] R. Klein, H. Antes, and D. Le Houédec, "Efficient 3D modelling of vibration isolation by open trenches," Computers and Structures, vol. 64, no. 1-4, pp. 809-817, 1997.

[16] H. Takemiya, K.-S. Shim, and K. Goda, "Embankment train track on soil stratum and wave impeding block (WIB) measured for vibrations reduction," in Soil Dynamics and Earthquake Engineering VII, C. A. Brebbia and A. S. Cakmak, Eds., vol. 14, pp. 105-112, Wessex Institute of Technology, Wessex, UK; Princeton University, Princeton, NJ, USA, 1995. 
[17] K. R. Massarsch, "Mitigation of traffic-induced ground vibration," in Proceedings of the 11th International Conference on Soil Dynamics and Earthquake Engineering, Berkeley, Calif, USA, January 2004.

[18] G. Lanzo and F. Silvestri, Risposta Sismica Locale. Teorie ed Esperienze, Hevelius, 1999.

[19] L. Andersen and C. J. C. Jones, "Coupled boundary and finite element analysis of vibration from railway tunnels-a comparison of two- and three-dimensional models," Journal of Sound and Vibration, vol. 293, no. 3-5, pp. 611-625, 2006.

[20] C. M. Di Liberto and G. Di Mino, "I fenomeni vibrazionali generati dal transito dei vettori ferroviari: analisi FEM del comportamento meccanico del sistema sovrastrutturaterreno. Casi studio per configurazione in trincea," Ingegneria Ferroviaria, pp. 53-62, 2006.

[21] G. Di Mino, "Le vibrazioni in campo ferroviario: analisi F.E.M. dell'interazione convoglio-sovrastruttura-terreno," in Proceedings of the 13th Convegno Nazionale SIIV, Padova, Italy, October 2003.

[22] C. J. C. Jones and J. R. Block, "Prediction of ground vibration from freight trains," Journal of Sound and Vibration, vol. 193, no. 1, pp. 205-213, 1996.

[23] V. Aiello and F. Silvestri, "Vibrazioni prodotte da infrastrutture di trasporto in ambiente urbano: una proposta metodologica applicata ad un caso studio," in Proceedings of the 22nd Convegno Nazionale di Geotecnica, Palermo, Italy, Sebtember 2004.

[24] L. Hall, "Simulations and analyses of train-induced ground vibrations in finite element models," Soil Dynamics and Earthquake Engineering, vol. 23, no. 5, pp. 403-413, 2003.

[25] M. H. El Naggar and A. G. Chehab, "Vibration barriers for shock-producing equipment," Canadian Geotechnical Journal, vol. 42, no. 1, pp. 297-306, 2005.

[26] R. Courant, K. Friedrichs, and H. Lewy, "On the partial difference equations of mathematical physics," IBM Journal, vol. 11, pp. 215-234, 1967.

[27] A. Castellani, "Boundary conditions to simulate an infinite space," Meccanica, vol. 9, no. 3, pp. 199-205, 1974.

[28] J. Lysmer and R. L. Kuhlemeyer, "Finite dynamic model for infinite media," Journal of the Engineering Mechanics Division, vol. 95, pp. 859-875, 1969.

[29] G. Di Mino, C. M. Di Liberto, and J. Nigrelli, "A FEM model of rail track-ground system to calculate the ground borne vibrations: a case of rail track with wooden sleepers and $\mathrm{k}$ fastenings at Castelvetrano," in Advanced Characterisation of Pavement and Soil Engineering Materials, A. Loizos, T. Scarpas, and I. Al-Qadi, Eds., Taylor \& Francis, London, UK, 2007.

[30] S. Haykin, NN: A Comprehensive Foundation, Macmillan, New York, NY, USA, 1994.

[31] J. C. Principe, N. R. Euliano, and C. W. Lefebvre, Neural and Adaptive Systems: Fundamentals Through Simulations, John Wiley \& Sons, New York, NY, USA, 2000. 

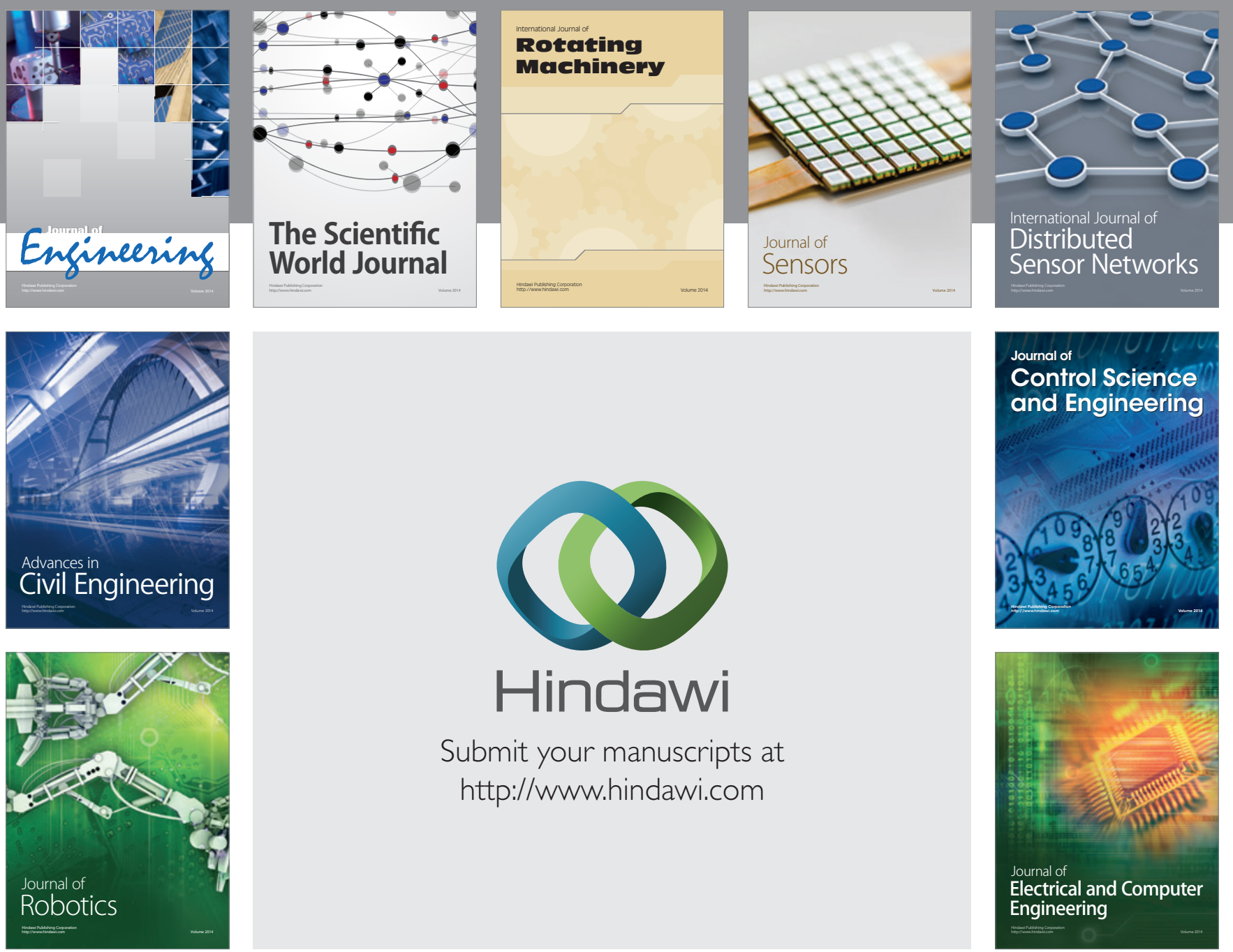

Submit your manuscripts at

http://www.hindawi.com
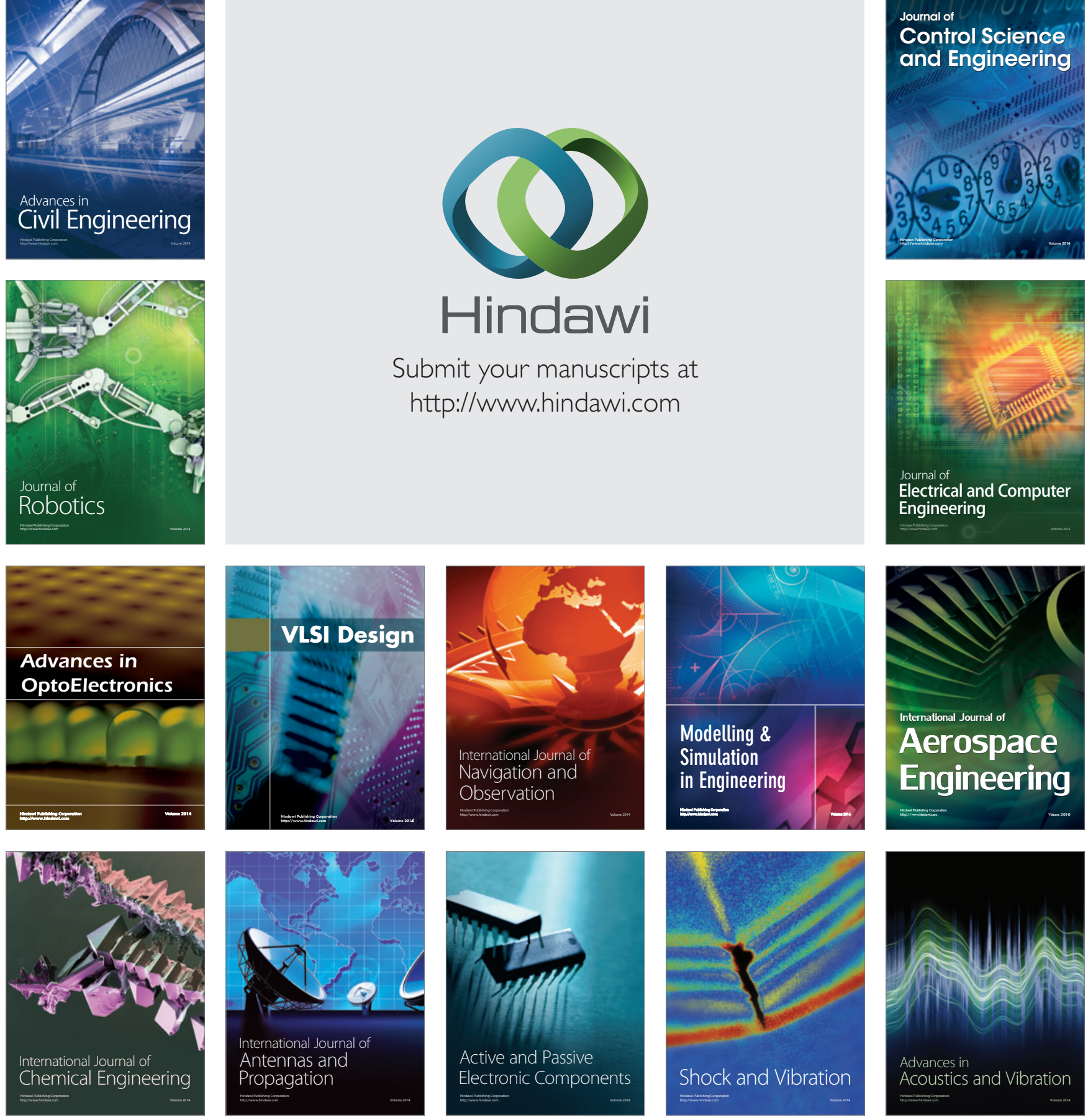